\title{
STUDIES OF ORGANIC MOLECULES CONTAINING METHYL GROUPS IN DARK CLOUDS
}

\author{
P. Friberg, W.M. Irvine, S.C. Madden ${ }^{2}$ A. Hjalmarson ${ }^{1}$ \\ 1 Onsala Space Observatory, S-439 00 Onsala, Sweden \\ 2 FCRAO, Univ. of Massachusetts, Amherst, MA 01003, USA
}

We have observed transitions of methano1, methyl acetylene (1), (2) and acetaldehyde $(3)$ in dark clouds. In methanol the $20_{0}^{-1} 0_{0} \mathrm{~A}^{+}, 1_{0}-0_{0} \mathrm{~A}^{+}$, $2_{-1}-1_{-1} E$ lines and tentatively the $20-10 \mathrm{E}$ line have been observed. The $1_{0}-0_{0} \mathrm{E}, 2_{1}-1_{1} \mathrm{E}$ and the $0_{0}-1_{-1} \mathrm{E}$ lines were searched for but not detected. Maps of TMC $1, L 134 \mathrm{~N}$, and B335 in the $20_{0}^{-1} \mathrm{~A}^{+}$and $2_{-1}^{-1}-1 \mathrm{E}$ lines show that the methanol emission is very extended and follows the extent of the $\mathrm{C}^{18} 0 \mathrm{~J}=1-0$ emission we11.

In TMC1 the methano1 emission extends over $25^{\prime}$ along the ridge, covering the ammonia and cyanopolyyne $\left(\mathrm{HC}_{2 * n+1}, \mathrm{n}=1,2,3, \ldots\right)$ emission. However, all of these species peak at different positions along the ridge, with the methanol maximum $6^{\prime} \mathrm{W}$ and $9^{\prime} \mathrm{N}$ of the cyanopolyyne peak. Further, the methanol emission in TMC1 has a velocity structure that reveals several clumps in the cloud.

The same general features are apparent in $\mathrm{L} 134 \mathrm{~N}$. The methanol emission follows the $\mathrm{C}^{18} 0$ emission and not that of ammonia nor ions such as $\mathrm{H}^{13} \mathrm{CO}^{+}$. B335 is too small to allow any comparison of the distribution of different species.

In TMC 1 the $\mathrm{CH}_{3} \mathrm{CCH}$ emission follows the cyanopolyyne distribution and not that of methanol nor $\mathrm{C}^{18} 0$. This suggests that molecules containing methyl groups fall into two different classes: i) carbon chains with a methyl group attached $i i)$ saturated molecules with methyl groups. The former class follows the distribution of the cyanopolyynes while the latter follows the distribution of molecules such as $\mathrm{C}^{18} 0$. Our detection of acetaldehyde, with a similar column density in both TMC1 and $\mathrm{L} 134 \mathrm{~N}\left(\mathrm{~N} \sim 6 * 10^{12} \mathrm{~cm}^{-2}\right)$ supports this theory.

The ratios of the intensities of methanol lines in TMC 1 te 11 us that the excitation is not in LTE. In the $\mathrm{E}$ species of methanol the energy levels in the $k=0$ ladder are underpopulated relative to the $k=-1$ ladder. The ratio of the intensities of the $2_{0}-1_{0} \mathrm{~A}^{+}$and the $1_{0}-0_{0} \mathrm{~A}^{+}$ is lower than expected in LTE in the optically thin limit. We derive $\mathrm{N} \sim 4 * 10^{13} \mathrm{~cm}^{-2}$ for TMC1 which is accurate within a factor of two or three. The non-LTE excitation complicates the derivation of the abundance ratio between the $A$ and $E$ species of methano1. We find a value between 1 and 2 for the ratio of $\mathrm{A} / \mathrm{E}$. The column density in L134N is, within the uncertainty, the same as TMC1 (c.f. acetaldehyde), while 
B335 has a column density of approximately $2 * 10^{13} \mathrm{~cm}^{-2}$.

In methyl acetylene the $\mathrm{J}_{\mathrm{k}}=5_{0}-40,5_{1}-41$ lines have been observed while we fail to detect the $\mathrm{J}_{\mathrm{k}}=5_{2}-42$ line. At low temperatures the ratio of antenna temperatures between the $k=1$ and $k=2$ components is a sensitive function of temperature. This since the $J_{k}=5_{1}$ state has an energy of $11.5 \mathrm{~K}$ and the $\mathrm{J}_{\mathrm{k}}=5_{2}$ state an energy of $32.4 \mathrm{~K}$ above the lowest para state (i.e. above $J_{k}=1_{1}$ ). The observed ratio of greater than or equal to ten, in TMC1, gives a kinetic temperature of $10 \mathrm{~K}$ according to statistical equlibrium calculations. The column density of methyl acetylene at the peak position in TMC1 is about $1 * 10^{14} \mathrm{~cm}^{-2}$. So methyl acetylene is roughly twice as abundant as methanol at the carbonchain peak.

Also our failure to detect the $2_{1}-1_{1} E$ line and the only tentative detection of the $20-10 \mathrm{E}$ line of methanol gives a low temperature estimate. The energy of the $2_{1}$ and $2_{0}$ states are $20.1 \mathrm{~K}$ and $12.2 \mathrm{~K}$ above the ground E-state $(1-1)$, respectively. The methanol excitation appears non-LTE but an "average" rotation temperature is about $5 \mathrm{~K}$ in dark clouds.

Over the area in TMC1 where both methanol and methyl acetylene has been observed they do show emission from the same cloud components. However, it seems that several cloud components fail to show any methyl acetylene emission. This occurs in the NW part of the ridge.

\section{References}

Irvine, W.M., Hoglund, B., Friberg, P., Askne, J., E11dér, J.: 1981, Ap. J. (Letters), 248, L113.

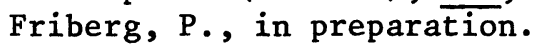

Matthews, H.E., Friberg, P., Irvine, W.M.: 1985, Ap. J., in press. 